\title{
On the average secrecy capacity for indoor visible light communication systems
}

\author{
Jun Zheng Zhang ${ }^{\dagger}$ and $\mathrm{Ke} \mathrm{Ke}^{* \dagger}$ (D)
}

\begin{abstract}
For visible light communication (VLC), the light signals are transmitted without optical fibers or any sort of wave-guiding. Due to the inherent broadcast nature, physical-layer security emerges as a promising method to protect information delivery from eavesdropping. As for the secrecy capacity of VLC channel, there exist two features. In one way, the limited optical power makes the common capacity expressions in radio-frequency (RF) communication unapplicable for VLC. In another way, several correlated geometrical parameters directly alters the Lambertian model of indoor VLC channel, which gives the secrecy capacity more meanings. However, the issue considering both aspects has not been studied recently. In this paper, from the practical scenarios, we extract a typical geometrical model to reveal the mobility principles of the legitimate receiver and the eavesdroppers. Then, we character two typical distributions of the geometrical parameter. Correspondingly, we derive the upper and lower bounds on the average secrecy capacity, which have the closed forms. Finally, simulation results show that our upper and lower bounds are tight at high optical signal-to-noise rates (OSNRs). Moreover, the geometrical features of VLC systems and distribution parameters of the receiver mobility are effectively reveal by the bounds.
\end{abstract}

Keywords: Visible light communication, Geometrical property, Average secrecy capacity, Upper and lower bounds

\section{Introduction}

In recent years, because of the light-emitting diodes (LEDs) widely deployed for energy conservation in practical illumination, visible light communication (VLC) is currently considered as one of the most indispensable green communication technologies and has attracted considerable attention [1-3]. For VLC, despite LOS propagation and better signal confinement, the light signals are transmitted without optical fibers or any sort of wave-guiding. The inherent broadcast nature makes VLC links inherently susceptible to eavesdropping by unintended or unauthorized users having access to the physical area illuminated by the data transmitters. Typical scenarios include public areas such as classrooms, offices, libraries, cafes and so on. Therefore, physical-layer security emerged as a promising method [4] to complement

\footnotetext{
*Correspondence: vlckeke@aliyun.com

† Jun Zheng Zhang and Ke Ke contributed equally to this work.

National Digital Switching System Engineering \& Technological Research Center, 450000 Zhengzhou, China
}

conventional encryption techniques and provide a first line of defense against eavesdropping attacks.

By contrast to radio-frequency (RF) communication, there exist two main features in the secrecy capacity. Intensity modulation with direct detection (IM/DD), which modulates the input data into the light beams emitted from LEDs [5-7], is always employed in indoor VLC system.

Feature 1: The optical intensity signals are limited to be a nonnegative real variable and ought to be less than the eye-safety standard[5, 6]. So, Gaussian distribution is not necessarily followed by the capacity-achieving input distribution [8], thus making the secrecy capacity expressions in RF communication not suitable for VLC.

Feature 2: Compared with the log-normal or Gaussian distribution, the Lambertian is often assumed to be a model of indoor VLC channel, which directly changes with several geometrical parameters, relating to the transmitters and receivers spatial locations [5-7]. Obviously,
Springer Open

(c) The Author(s). 2020 Open Access This article is licensed under a Creative Commons Attribution 4.0 International License, which permits use, sharing, adaptation, distribution and reproduction in any medium or format, as long as you give appropriate credit to the original author(s) and the source, provide a link to the Creative Commons licence, and indicate if changes were made. The images or other third party material in this article are included in the article's Creative Commons licence, unless indicated otherwise in a credit line to the material. If material is not included in the article's Creative Commons licence and your intended use is not permitted by statutory regulation or exceeds the permitted use, you will need to obtain permission directly from the copyright holder. To view a copy of this licence, visit http://creativecommons.org/licenses/by/4.0/. 
the geometrical feature give the secrecy performance of VLC channels more meanings.

In [9], Shannon pioneered the framework of information-theoretic security. Wyner in [10] proposed a fundamental infromation-theoretic security measure, termed as the secrecy capacity. Motivated by Wyner's work, many studies [11-16] on the secrecy performance of Gaussian wiretap channels were considered. However, despite that there exist many studies on the secrecy performance for RF communication, the corresponding study for VLC channel is rare.

As for IM/DD channels, Shannon referred to the challenge in deriving an analytical capacity expression for peak-limited channels [17]. In [8], the upper and lower capacity bounds were derived with the additive Gaussian channel, while a discrete Gaussian distribution is utilized in [18] to research the problem [19-22] studied the capacity bounds of optical wireless channels. Furthermore, as for the secrecy performance, [23] derived the lower and upper bounds on the secrecy capacity of the scalar Gaussian wiretap channel subject to amplitude constraints. Mukherjee [24] analyzed the secret-key transmission strategy of multiple-input single-output (MISO) VLC systems after deriving the upper and lower bounds. Shen et al. [25] employed transmit beamforming and jamming techniques to enhance the secrecy for MISO-VLC systems. However, they all didn't consider the geometrical property of indoor VLC systems. Particularly, although [26] derive the closed-form analytical expressions for secrecy outage probability and the average secrecy capacity, the study was based on the secrecy capacity expression of Gaussian channel. From the above, the issue considering both features has not been studied currently.

Because of the above-mentioned factors, in this paper, we research the relation between the secrecy capacity with the geometrical property, based on the power-limited channel capacity. Particularly, we extract a typical geometrical model from the practical scenarios, which reveals the mobility principles of the legitimate receiver and the eavesdroppers. Then, we character two typical distributions of the geometrical parameter. Accordingly, the upper and lower bounds of the average secrecy capacity are derived, which have the closed forms and are related to the geometrical parameters of VLC systems and distribution parameters of the receiver mobility. Finally, simulation results show that our upper and lower bounds are tight at high OSNRs and the geometrical feature are effectually revealed.

For conciseness, the key notations throughout the paper are summarized in the following Table 1.

\section{System model}

In this paper, a typical indoor VLC system is considered, which with a LED as the transmitter and a photo-detector
Table 1 Key notations used in this paper

\begin{tabular}{ll}
\hline Notations & Explanation \\
\hline $\mathcal{C}_{s}$ & Secrecy capacity \\
$\overline{\mathcal{C}}_{s}$ & Average secrecy capacity \\
$A$ & Peak power \\
$\varepsilon$ & Average power \\
$\alpha$ & Optical average-to-peak power ratio \\
$\mathbb{E}_{x}(\cdot)$ & Mean value of a variable to $x$. \\
$\phi$ & Emergence angle \\
$\psi$ & Incidence angle \\
$m$ & Lamberts mode order \\
$\Phi_{\frac{1}{2}}$ & Transmitter half-power semiangle \\
$\mathcal{R}$ & Illumination region \\
$l$ & Vertical distance \\
$d_{i}$ & Receiver and center distance \\
$O_{i}$ & Center of $d_{i}$ mobility region \\
$u_{i}$ & Radius of $d_{i}$ mobility region \\
$\zeta_{i}$ & Standard deviation of Gaussian distribution \\
$\left(x_{i}, y_{i}\right)$ & Coordinate of the receiver \\
$f\left(d_{i}\right)$ & The PDF of $d_{i}$ \\
$t_{i}$ & Distance of $O_{i}$ and center \\
$t_{i 1}, t_{i 2}$ & Coordinate of $O_{i}$ \\
\hline
\end{tabular}

as a legitimate receiver. Moreover, there exist some eavesdroppers, which locate in the same communication region and try to eavesdrop the information between the LED and the legitimate receiver. As shown in Fig. 1, the scenario is common, such as the office, the classroom, the airport lounge and so on.

The signals received by the legitimate receiver and the $i$ th $(i \geq 1)$ eavesdroppers are given by

$$
\begin{aligned}
& Y=h_{0} X+w_{0}, \\
& Z_{i}=h_{i} X+w_{i},
\end{aligned}
$$

where $w_{0}, w_{i} \sim \mathcal{N}_{\mathbb{R}}\left(0, \sigma^{2}\right)$ denote the additive Gaussian noise. For simplicity, let us denote the legitimate receiver as the 0 th receiver.

1. Optical Power Constraint. For illumination and eyesafety consideration, the optical average power $\varepsilon$, the input signal $X$, and peak power $A$ are constrained as

$$
\operatorname{Pr}(0 \leq X \leq A)=1,
$$

$$
\mathbb{E}[X] \leq \varepsilon,
$$

$X \in \mathbb{R}_{0}^{+}$. The optical average-to-peak power ratio (APPR) is defined as

$$
\alpha \triangleq \frac{\varepsilon}{A}
$$




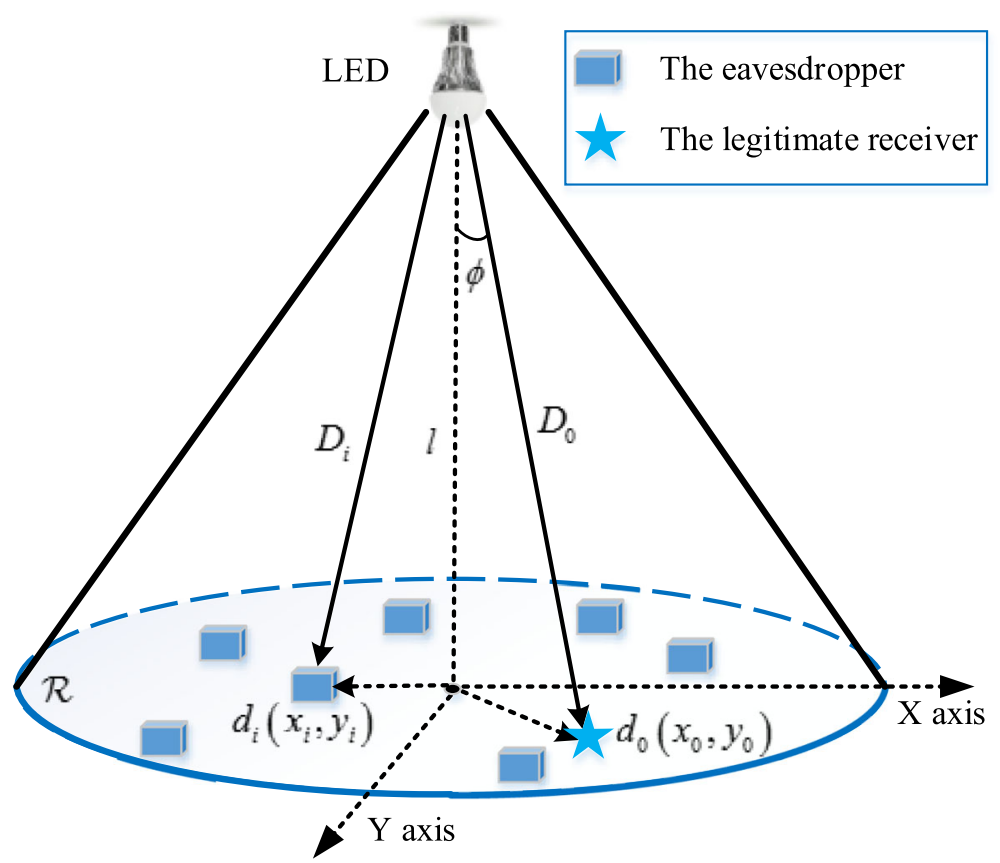

Fig. 1 The sketch map of a typical indoor VLC system and the Lambertian model parameters expression

where $0<\alpha \leq 1$.

2. Geometrical Property. Although RF channel often considers multipath effect, line-of-sight (LOS) path is often assumed to exist in the typical indoor point-to-point channel. The channel gain $h_{i},(i \geq 0)$ is derived by the Lambertian model $[8,9]$ as

$$
h_{i}=\left\{\begin{array}{l}
\frac{S_{r}(m+1)}{2 \pi D_{i j}^{2}} \cos ^{m}(\phi) \cos (\psi), 0 \leq \psi \leq \Psi_{c}, \\
0, \psi>\Psi_{c},
\end{array}\right.
$$

where $\phi$ is the emergence angle of transmitter and $\psi$ being the incidence angle of receiver. $S_{r}$ is the detector acreage, $D_{i j}$ denotes the distance between the transmitter and receiver, and $\Psi_{c}$ is the detector field-of-view (FOV) semiangle. $\Phi_{\frac{1}{2}}$ is the transmitter half-power semiangle. The Lamberts mode order is derived by

$$
m=-\frac{\ln (2)}{\ln \left(\cos \left(\Phi_{\frac{1}{2}}\right)\right)} .
$$

In this paper, let $\phi=\psi$ and meanwhile $\cos (\phi)=$ $\cos (\psi)=\frac{l}{D}$. Additional, (5) can be rewritten as

$$
h_{i}=\frac{S_{r}(m+1) l^{(m+1)}}{2 \pi\left(l^{2}+d_{i}^{2}\right)^{\frac{m+3}{2}}},(i \geq 0) .
$$

When scenario is given, the $S_{r}, m$ being fixed, $h$ is related to the movement of the receiver, calculated by $l$ and $d_{i}$.
$M=l \cdot \tan \Phi_{\frac{1}{2}}$ represents the maximum value of $d$, which is the radius of illumination region $\mathcal{R}$. Here, The distribution of $d_{i}$ under the given $l$ mainly attract our attention.

$$
d_{i}^{2}=x_{i}^{2}+y_{i}^{2},(i \geq 0)
$$

where $x_{i}, y_{i}$ are the coordinate values.

According to the distinct features of VLC channel, our problem can be summarized as follows:

\section{Problem 1:}

Considering the geometrical property and the optical power constraint, how to derive the closed-form expression for average secrecy capacity. The mathematical model involved is given as ([27])

$$
\begin{aligned}
& \overline{\mathcal{C}_{s}}=\mathbb{E}_{h_{0}, h_{i}}\left[\mathcal{C}_{s}\right] \\
& =\mathbb{E}_{h_{0}, h_{i}}\left[\max _{p_{X}}(\mathbb{I}(X ; Y)-\mathbb{I}(X ; Z))\right] \\
& \text { s.t. } \quad\left\{\begin{array}{l}
\operatorname{Pr}(0 \leq X \leq A)=1 \\
\mathbb{E}[X] \leq \varepsilon
\end{array}\right.
\end{aligned}
$$

Combining (7) and (9), the average secrecy capacity $\overline{\mathcal{C}}_{s}$ of the legitimate receiver with the $i$ th $(i \geq 1)$ eavesdropper can be given as 


$$
\begin{aligned}
\overline{\mathcal{C}}_{s}\left(h_{0}, h_{i}\right) & =\mathbb{E}_{h_{0}, h_{i}}\left[\mathcal{C}_{s}\left(h_{0}, h_{i}\right)\right] \\
& =\mathbb{E}_{d_{0}, d_{i}}\left[\mathcal{C}_{s}\left(d_{0}, d_{i}\right)\right] \\
& =\iint_{\mathcal{S}_{0}} \iint_{\mathcal{S}_{i}} \mathcal{C}_{s}\left(d_{0}, d_{i}\right) f\left(d_{0}, d_{i}\right) \mathrm{d} \tau_{0} \mathrm{~d} \tau_{i} \\
& \stackrel{\left(c_{0}\right)}{=} \iint_{\mathcal{S}_{0}} \iint_{\mathcal{S}_{i}} \mathcal{C}_{s}\left(d_{0}, d_{i}\right) f\left(d_{0}\right) f\left(d_{i}\right) \mathrm{d} \tau_{0} \mathrm{~d} \tau_{i},
\end{aligned}
$$

where $\mathcal{C}_{s}\left(d_{0}, d_{i}\right)$ is analyzed in (11), (12) and (13). The term $f\left(d_{0}, d_{i}\right)$ is the joint probability density function (PDF) and the terms of $f\left(d_{0}\right), f\left(d_{i}\right)$ represent the indoor human mobility, respectively. Without loss of generality, we assume that the mobilities of the eavesdroppers and the legitimate receiver are independent with each other. Thus, we have $f\left(d_{0}, d_{i}\right)=f\left(d_{0}\right) f\left(d_{i}\right)$, making the transformation of $\left(c_{0}\right)$.

\section{Average secrecy capacity}

Based on (10), we firstly analyze the bounds on the secrecy capacity $\mathcal{C}_{s}$. Then, by characterising the geometrical property of the practical scenarios, we extract two typical distribution model of $d_{i},(i \geq 0)$, which is related to $h_{i},(i \geq$ 0 ). Further, we correspondingly derive the lower bounds on the average secrecy capacity.

\section{Bounds on secrecy capacity $\mathcal{C}_{s}$}

Based on the definition of capacity [28], due to the constraints (2) and (3) for VLC, the capacity-achieving input distribution does not always follow Gaussian distribution [8]. Thus, the common Shannon formula in RF communication can not be applied in VLC.

According to [23], the secrecy capacity of the legitimate receiver with the $i$ th $(i \geq 1)$ eavesdropper is lower-bounded by each of the following two bounds

$$
\begin{aligned}
\mathcal{C}_{s} \geq & \frac{1}{2} \log \left(1+\frac{2 h_{0}^{2} A^{2}}{\pi e \sigma^{2}}\right) \\
& -\left(1-2 \mathcal{Q}\left(\frac{\delta+h_{i} A}{\sigma}\right)\right) \log \frac{2\left(h_{i} A+\delta\right)}{\sqrt{2 \pi \sigma^{2}}\left(1-2 \mathcal{Q}\left(\frac{\delta}{\sigma}\right)\right)} \\
& -\mathcal{Q}\left(\frac{\delta}{\sigma}\right)-\frac{\delta}{\sqrt{2 \pi \sigma^{2}}} e^{-\frac{\delta^{2}}{2 \sigma^{2}}}+\frac{1}{2}, \\
\mathcal{C}_{s} & \geq \frac{1}{2} \log \frac{6 h_{0}^{2} A^{2}+3 \pi e \sigma^{2}}{\pi e h_{i}^{2} A^{2}+3 \pi e \sigma^{2}},
\end{aligned}
$$

and is upper-bounded by

$$
\mathcal{C}_{s} \leq \frac{1}{2} \log \frac{h_{0}^{2} A^{2}+\sigma^{2}}{h_{i}^{2} A^{2}+\sigma^{2}} .
$$

Note that as analyzed in [23], the lower bounds and upper bounds on the secrecy capacity are tight at asymptotically high SNR. Where $\delta$ is a free parameter such that $\delta>0$ and $\sigma^{2}$ is variance of the additive Gaussian noise. Other parameter is metioned above. Due to that (12) and (13) have the simpler expression, we will utilize them to analyze the average secrecy capacity in the following.

\section{Mobility model of $d_{i}$}

Here, we firstly model the geometrical distribution of typical scenarios. For the practical scenarios such as the classroom, the airport lounge and so on, we tend to move within a relatively region. Without loss of generality, let us assume that each receiver locates at a relatively-fixed region.

Figure 2 shows the sketch of our mobility model. The $i$ th $(i \geq 0)$ receiver moves within the corresponding circle with the radius $u_{i} . t_{i}$ is the distance between the center of the circle $O_{i}$ to the center of illumination region. We use a 3-D cartesian coordinate system at the receivers height to identify their locations. The origin $(0,0)$ corresponds to the room center. Then, we have

$$
\begin{aligned}
& 0 \leq\left(x_{i}-t_{i 1}\right)^{2}+\left(y_{i}-t_{i 2}\right)^{2} \leq u_{i}^{2}, \\
& x_{i}^{2}+y_{i}^{2}=d_{i}^{2}, \\
& t_{i 1}^{2}+t_{i 2}^{2}=t_{i}^{2} .
\end{aligned}
$$

where $t_{i 1}$ and $t_{i 2}$ are coordinate of $O_{i}$. Although our human mobility is various from person to person, there exist some basic principles. Here two typical mobility types are mainly proposed as follows:

- Type of main location. This means that the possibility of sitting in a main location is often much larger than the surrounding area. In the practical restaurant, cafes and so on, we tend to sit at the desks or the other relatively-fixed location. Typically, as for the $i$ th receiver, we model $f\left(d_{i}\right)$ in (10) as the 2 -dimension Gaussian distribution at the receiver height, i.e.,

$$
f\left(d_{i}\right)=\frac{1}{2 \pi \zeta_{i 1} \zeta_{i 2}} e^{-\frac{\left(x_{i}-t_{i 1}\right)^{2}}{2 \zeta_{i 1}^{2}}-\frac{\left(y_{i}-t_{i 2}\right)^{2}}{2 \zeta_{i 2}^{2}}},(i \geq 0)
$$

Note that it is simple to extend (15) to the specific human mobility, by adjusting $\zeta_{i 1}, \zeta_{i 2}, t_{i 1}, t_{i 2}$. Here, the other scenarios are based on a scenario of one LED and one main location. Furthermore, let us assume ${ }^{1}$ $\zeta_{i 1}=\zeta_{i 2}=\zeta_{i} \neq 0$. Then, (15) can be transformed as

$$
f\left(d_{i}\right)=\frac{1}{2 \pi \zeta_{i}^{2}} e^{-\frac{\left(x_{i}-t_{i 1}\right)^{2}+\left(y_{i}-t_{i 2}\right)^{2}}{2 \zeta_{i}^{2}}},(i \geq 0) .
$$

\footnotetext{
${ }^{1}$ Here, we consider the simple case of $\zeta_{i 1}=\zeta_{i 2}$, which can be simply transformed to the other practical scenarios.
} 


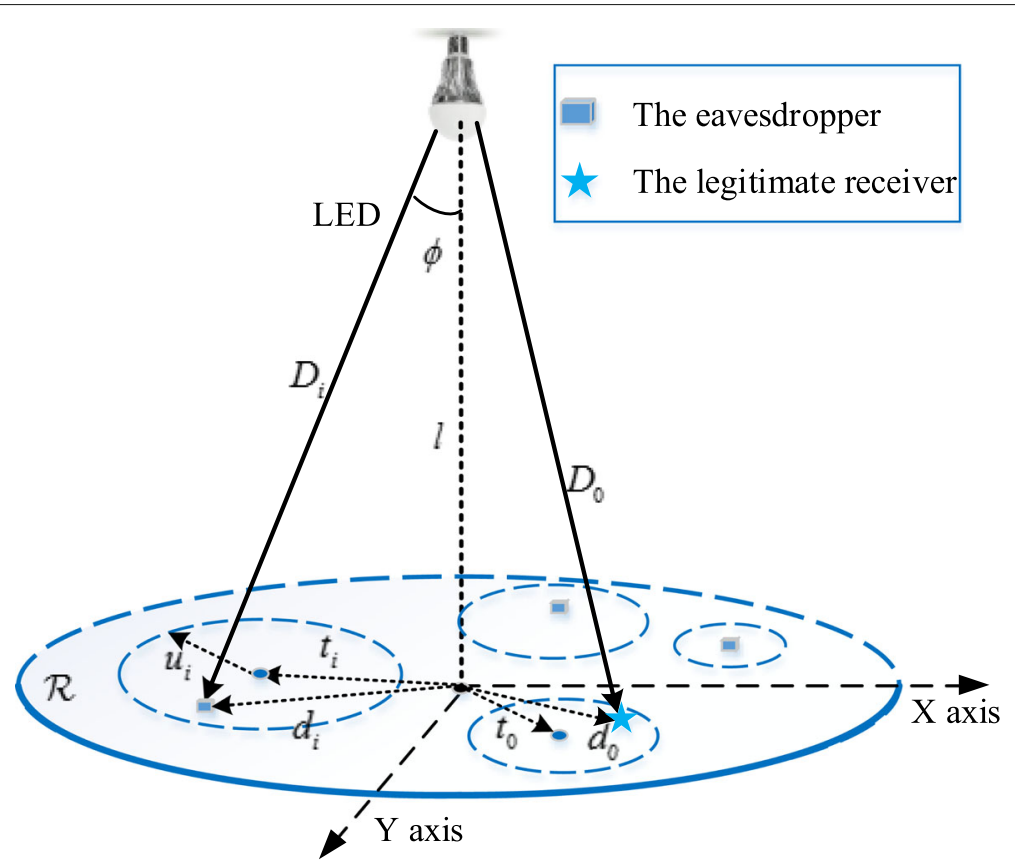

Fig. 2 The sketch map of a typical indoor VLC system and the parameters in Lambertian model

- Type of random walk. The meaning is that the possibilities of the receiver at any point within the circle are same. For example, we often randomly and steadyly walk in the relatively-fixed region. Typically, $f\left(d_{i}\right)$ is modeled as the uniform distribution, i.e.,

$$
f\left(d_{i}\right)=\frac{1}{\pi u_{i}^{2}},(i \geq 0)
$$

and the other random-walk scenarioswhich is based on it. For the practical random walk ${ }^{2}$, we can readily deduce the corresponding average secrecy capacity with the same manner as the case of (17).

For simplicity, let us make some notations.

$$
T_{1} \triangleq \frac{S_{r}(m+1) l^{m+1}}{2 \pi}, T_{2} \triangleq \frac{m+3}{2} .
$$

For the legitimate receiver and the $i$ th $(i \geq 1)$ eavesdropper,

$$
\begin{aligned}
& h_{0 L} \triangleq T_{1}\left(l^{2}+t_{0}^{2}\right)^{-T_{2}}, \\
& h_{0 R} \triangleq T_{1}\left(l^{2}+\left(t_{0}+u_{0}\right)^{2}\right)^{-T_{2}}, \\
& h_{i L} \triangleq T_{1}\left(l^{2}+\left(t_{i}-u_{i}\right)^{2}\right)^{-T_{2}}, \\
& h_{i R} \triangleq T_{1}\left(l^{2}+t_{i}^{2}\right)^{-T_{2}} .
\end{aligned}
$$

And then, the following Theorem 1 illuminates the results for the average secrecy capacity, Appendix A illuminates the proof of which.

\footnotetext{
${ }^{2}$ Although our mobility may not be the whole region $\mathcal{R}$, there only exists a change of the integration region in (10).
}

For indoor VLC system, the upper bounds on the average secrecy capacity under different geometrical model are given as follows:

- 1) When $f\left(d_{i}\right)=\frac{1}{2 \pi \zeta_{i}^{2}} e^{-\frac{\left(x_{i}-t_{i 1}\right)^{2}+\left(y_{i}-t_{i 2}\right)^{2}}{2 \zeta_{i}^{2}}} \cdot \overline{\mathcal{C}}_{s}$ is upper-bounded by

$$
\overline{\mathcal{C}}_{s} \leq \frac{\left(1-e^{-\frac{u_{0}^{2}}{2 \zeta_{0}^{2}}}\right)\left(1-e^{-\frac{u_{i}^{2}}{2 \zeta_{i}^{2}}}\right)}{4} \mathcal{G}_{1}\left(u_{0}, t_{0}, u_{i}, t_{i}\right),
$$

$\overline{\mathcal{C}}_{s}$ is lower-bounded by one of the following bounds

$$
\overline{\mathcal{C}}_{s} \geq \frac{\left(1-e^{-\frac{u_{0}^{2}}{2 \zeta_{0}^{2}}}\right)\left(1-e^{-\frac{u_{i}^{2}}{2 \zeta_{i}^{2}}}\right)}{4} \mathcal{G}_{2}\left(u_{0}, t_{0}, u_{i}, t_{i}\right),
$$

$$
\begin{aligned}
\overline{\mathcal{C}}_{s} \geq & \left(1-e^{-\frac{u_{0}^{2}}{2 \zeta_{0}^{2}}}\right)\left(1-e^{-\frac{u_{i}^{2}}{2 \zeta_{i}^{2}}}\right)\left[-p_{3}+\right. \\
& \left.\frac{p_{1}\left(h_{0 L}\right)+p_{1}\left(h_{0 R}\right)}{4}-\frac{p_{2}\left(h_{i L}\right)+p_{2}\left(h_{i R}\right)}{2}\right] .
\end{aligned}
$$


- 2)When $f\left(d_{i}\right)=\frac{1}{\pi u_{i}^{2}}$. $\overline{\mathcal{C}}_{s}$ is upper-bounded as

$$
\overline{\mathcal{C}_{s}} \leq \frac{1}{4} \mathcal{G}_{1}\left(u_{0}, t_{0}, u_{i}, t_{i}\right)
$$

$\overline{\mathcal{C}}_{s}$ is lower-bounded by one of the following bounds

$$
\begin{aligned}
& \overline{\mathcal{C}}_{s} \geq \frac{1}{4} \mathcal{G}_{2}\left(u_{0}, t_{0}, u_{i}, t_{i}\right), \\
& \overline{\mathcal{C}}_{s} \geq \frac{p_{1}\left(h_{0 L}\right)+p_{1}\left(h_{0 R}\right)}{4}-\frac{p_{2}\left(h_{i L}\right)+p_{2}\left(h_{i R}\right)}{2}-p_{3} .
\end{aligned}
$$

Among them, $p_{1}(\cdot), p_{2}(\cdot), p_{3}$ are defined in (54), (55) and (56). The terms of $\mathcal{G}_{1}\left(u_{0}, t_{0}, u_{i}, t_{i}\right), \mathcal{G}_{2}\left(u_{0}, t_{0}, u_{i}, t_{i}\right)$ are given in (26) and (27).

In Theorem 1,

$$
\begin{aligned}
\mathcal{G}_{1}\left(u_{0}, t_{0}, u_{i}, t_{i}\right)= & \log \left[\sigma^{2}+A^{2} T_{1}^{2}\left[l^{2}+\left(t_{0}-u_{0}\right)^{2}\right]^{-2 T_{2}}\right] \\
& +\log \left[\sigma^{2}+A^{2} T_{1}^{2}\left(l^{2}+t_{0}^{2}\right)^{-2 T_{2}}\right] \\
& -\log \left[\sigma^{2}+A^{2} T_{1}^{2}\left(l^{2}+t_{i}^{2}\right)^{-2 T_{2}}\right] \\
& -\log \left[\sigma^{2}+A^{2} T_{1}^{2}\left(l^{2}+\left(t_{i}+u_{i}\right)^{2}\right)^{-2 T_{2}}\right],
\end{aligned}
$$

$$
\begin{aligned}
\mathcal{G}_{2}\left(u_{0}, t_{0}, u_{i}, t_{i}\right)= & \log \left[3 \pi e \sigma^{2}+6 A^{2} T_{1}^{2}\left(l^{2}+t_{0}^{2}\right)^{-2 T_{2}}\right] \\
& +\log \left[3 \pi e \sigma^{2}+6 A^{2} T_{1}^{2}\left(l^{2}+\left(t_{0}+u_{0}\right)^{2}\right)^{-2 T_{2}}\right] \\
& -\log \left[3 \pi e \sigma^{2}+\pi e A^{2} T_{1}^{2}\left(l^{2}+t_{i}^{2}\right)^{-2 T_{2}}\right] \\
& -\log \left[3 \pi e \sigma^{2}+\pi e A^{2} T_{1}^{2}\left(l^{2}+\left(t_{i}-u_{i}\right)^{2}\right)^{-2 T_{2}}\right] \leftarrow
\end{aligned}
$$

\section{Simulation results}

In this section, the tightness and the geometrical feature of our upper and lower bounds on the average secrecy capacity are analyzed in Theorem 1 . We consider a scenario with several users under a LED as our simulation environment. $\frac{A}{\sigma}$ represents the OSNR. High OSNRs are the practical application zone, because of typical indoor illumination levels can offer at least $40 \mathrm{~dB}$ OSNR at the receiver [5]. Note that we use "Ratio" to express $\frac{u_{i}}{\zeta_{i}}$. Notation "U-B" denotes the simulation for the upper bound and Notation "L-B" for the lower bound.

Figure 3 shows the average secrecy capacity bounds under $l=1.5 \mathrm{~m}, 2.5 \mathrm{~m}, 3.5 \mathrm{~m}$, with $d_{i}$ yielding to the Gaussian distribution. Figure 4 plots the similar curves under different $\frac{u_{i}}{\zeta_{i}}=1,2,3$. Figure 5 shows that under different $t_{i}$ $=2.2 \mathrm{~m}, 5.2 \mathrm{~m}, 7.2 \mathrm{~m}$. As shown in figures, when $l$ increases, the average secrecy capacity degrades. When the distribution parameters of $\frac{u_{i}}{\zeta_{i}}, t_{i}$ increase, the average secrecy capacity can be improved. As shown in Theorem 1, the bounds are effective to reveal the geometrical features of VLC systems and distribution parameters of the receiver mobility.

Figure 6 shows the average secrecy capacity bounds under different $l$, with $d_{i}$ yielding to the uniform distribution. Figure 7 plots the similar curves under different $t_{i}$.In one way, we can observe that the same principle that when 1 increases, the average secrecy capacity degrades. When the distribution parameters of ti increase, the average secrecy capacity can be improved. In another way, by combing Fig. 3 with Figs. 5, 6 with Fig. 7, different PDF of

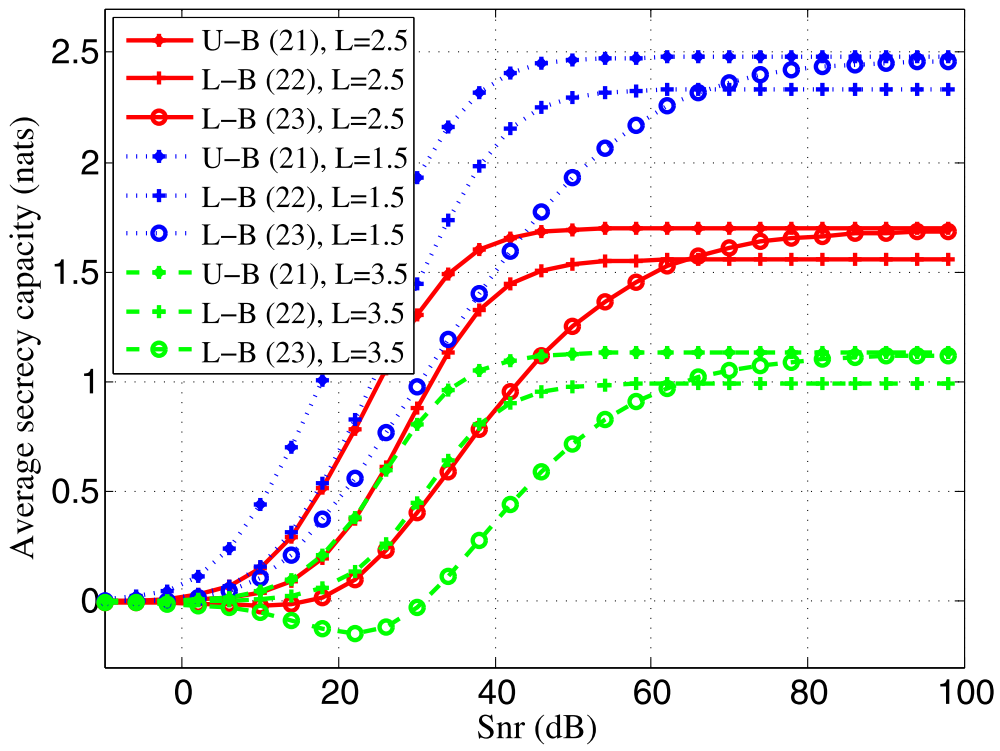

Fig. 3 Average secrecy capacity under different I, for Gaussian distribution 


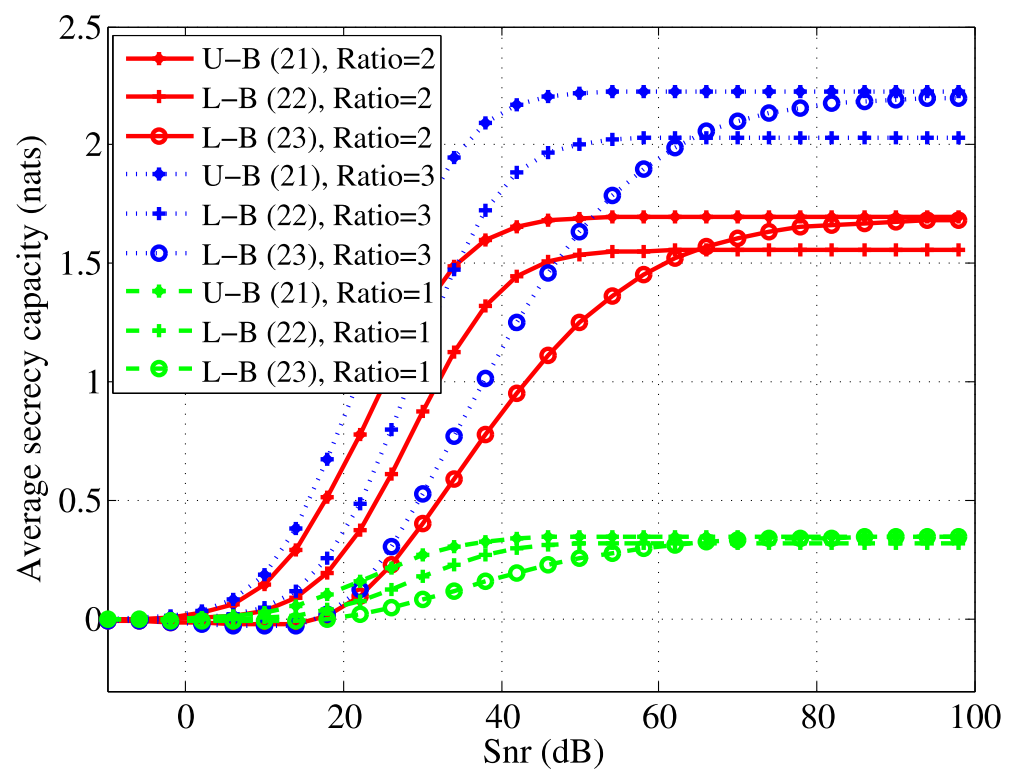

Fig. 4 Average secrecy capacity under various $\frac{u_{i}}{\zeta_{i}}$, for Gaussian distribution

the receiver mobility has different secrecy capacity even if under same parameters. This also reveals the influence of the geometrical property on the secrecy capacity, as shown in Theorem 1.

Moreover, according to Figs. 2, 3, 4 and 5, the lower bound in 22 keeps the relatively-stable gap with the upper bound in (21), while the lower bound in (23) has a relatively-varying gap. At the lower OSNRs, the former gap is smaller than the latter gap. At the higher OSNRs, the former gap is larger than the latter gap. Due to that typical indoor illumination levels can offer high OSNR at the receiver [5], high OSNRs are the practical application zone. From the results, under different conditions, our bounds just perform the better tightness at high OSNRs.

\section{Conclusion}

Due to the inherent broadcast nature of VLC channels, physical-layer security emerged as a promising method to

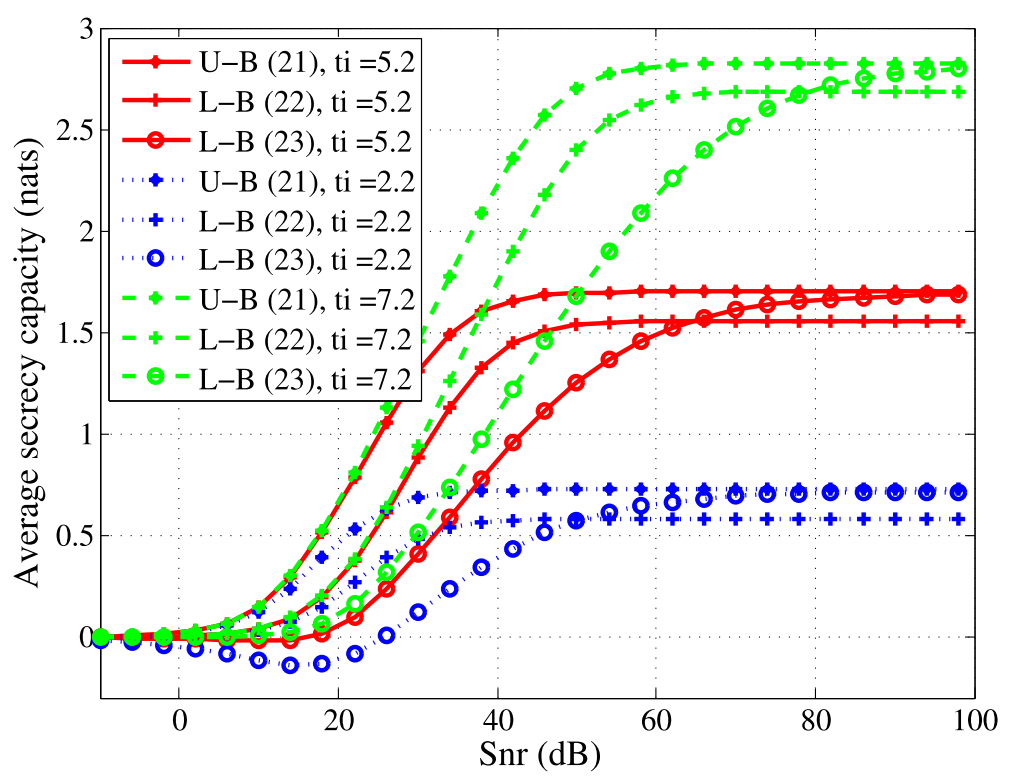

Fig. 5 Average secrecy capacity under different $t_{i}$, for Gaussian distribution 


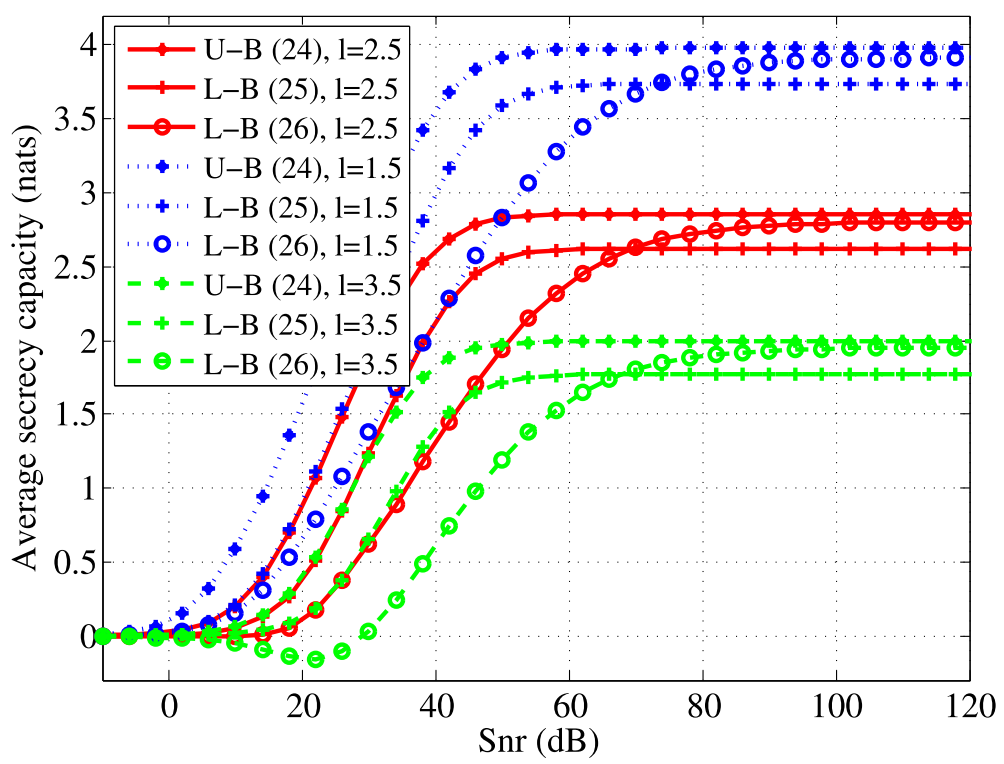

Fig. 6 Average secrecy capacity under different l, for uniform distribution

protect information delivery from eavesdropping. There exist two features within the secrecy capacity. The limited optical power makes the common capacity expressions in radio-frequency (RF) communication not suitable for VLC. In another way, the Lambertian model of indoor VLC channel directly changes with several related geometrical parameters, which makes the secrecy capacity more meanings. However, the study considering both aspects does not been carried out currently. This paper extracted a typical geometrical model from the practical scenario to reveal the mobility principles of the legitimate receiver and the eavesdroppers. Then, by characterizing two typical distributions of the geometrical parameter, we derived the corresponding upper- and lower- bounds on the average secrecy capacity, which have the closed forms. Simulation results reveal that our bounds are tight at high optical signal-to-noise rates (OSNRs). Moreover, the geometrical features of VLC systems and distribution parameters of the receiver mobility can be effectively revealed by the bounds.

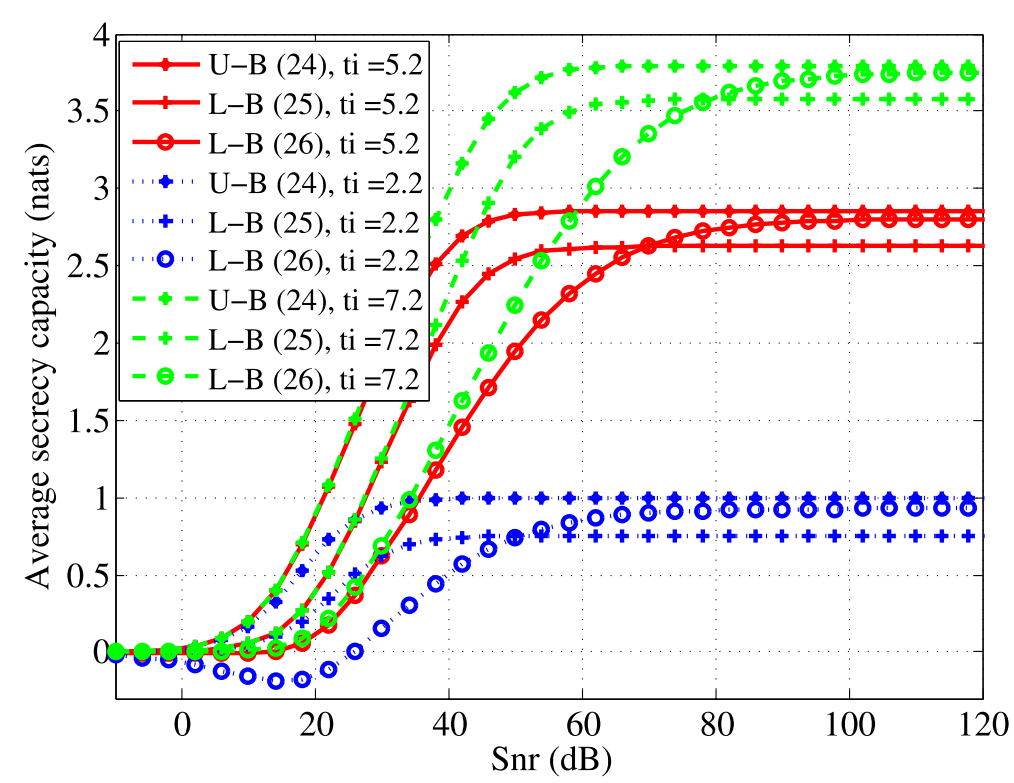

Fig. 7 Average secrecy capacity under different $t_{i}$, for Gaussian distribution 


\section{Appendix}

For simplicity, let

$$
h_{i}=T_{1}\left(l^{2}+d_{i}^{2}\right)^{-T_{2}}
$$

where $T_{1}=\frac{S_{r}(m+1) l^{m+1}}{2 \pi}, T_{2}=\frac{m+3}{2}$. For indoor VLC system, $m=-\frac{\ln (2)}{\ln \left(\cos \left(\Phi_{\frac{1}{2}}\right)\right)}>0$. Thus

$$
T_{1}>0, T_{2}>0 \text {. }
$$

Note that in (28) and (33), the subscript of $i=0$ is for the legitimate receiver, while the subscripts of $i \geq 1$ are for the eavesdroppers.

Here, we divide the circle $\mathcal{S}_{i}$, as shown in Fig. 8. For $i \in$ $[0, N]$, we have

$$
\begin{aligned}
& \mathcal{S}_{i}=\mathcal{S}_{i l}+\mathcal{S}_{i r} \\
& \iint_{\mathcal{S}_{i l}} f\left(d_{i}\right) \mathrm{d} \tau_{i}=\iint_{\mathcal{S}_{i r}} f\left(d_{i}\right) \mathrm{d} \tau_{i}=\frac{1}{2} \iint_{\mathcal{S}_{i}} f\left(d_{i}\right) \mathrm{d} \tau_{i},
\end{aligned}
$$

which means that the possibilities of the receiver $d_{i}\left(x_{i}, y_{i}\right)$ locating at $\mathcal{S}_{i l}, \mathcal{S}_{i r}$ are equal. When $d_{i}\left(x_{i}, y_{i}\right)$ lies in the halfcircle $\mathcal{S}_{i l}$, we have

$$
t_{i}-u_{i} \leq d_{i} \leq t_{i}
$$

When $d_{i}\left(x_{i}, y_{i}\right)$ lies in the half-circle $\mathcal{S}_{i r}$, we have

$$
t_{i} \leq d_{i} \leq t_{i}+u_{i}
$$

By using the polar coordinate system, let

$$
\begin{aligned}
& x_{i}=t_{i 1}+\rho_{i} \cos \theta_{i}, \\
& y_{i}=t_{i 2}+\rho_{i} \sin \theta_{i} .
\end{aligned}
$$

\section{Proof of upper bounds (20) and (23)}

We derive the upper bounds based on (13) and (10). Then, we have

$$
\begin{aligned}
& \overline{\mathcal{C}}_{s}\left(h_{B}, h_{E}\right)=\iint_{\mathcal{S}_{0}} \iint_{\mathcal{S}_{i}} \mathcal{C}_{s}\left(d_{0}, d_{i}\right) f\left(d_{0}\right) f\left(d_{i}\right) \mathrm{d} \tau_{0} \mathrm{~d} \tau_{i} \\
& \leq \iint_{\mathcal{S}_{0}} \iint_{\mathcal{S}_{i}} \frac{1}{2} \log \frac{h_{B}^{2} A^{2}+\sigma^{2}}{h_{E}^{2} A^{2}+\sigma^{2}} f\left(d_{0}\right) f\left(d_{i}\right) \mathrm{d} \tau_{0} \mathrm{~d} \tau_{i} \\
& =\frac{1}{2} \int \underbrace{\iint_{\mathcal{S}_{0}} \log \left(h_{B}^{2} A^{2}+\sigma^{2}\right) f\left(d_{0}\right) \mathrm{d} \tau_{0} \iint_{\mathcal{S}_{i}} f\left(d_{i}\right) \mathrm{d} \tau_{i}}_{w_{1}} \\
& -\frac{1}{2} \underbrace{\iint_{\mathcal{S}_{0}} f\left(d_{0}\right) \mathrm{d} \tau_{0} \iint_{\mathcal{S}_{i}} \log \left(h_{E}^{2} A^{2}+\sigma^{2}\right) f\left(d_{i}\right) \mathrm{d} \tau_{i}}_{w_{2}}
\end{aligned}
$$

Based on (28), the terms of $w_{1}, w_{2}$ are given by

$$
\begin{gathered}
w_{1}=\iint_{\mathcal{S}_{0}} \log \left[\sigma^{2}+A^{2} T_{1}^{2}\left(l^{2}+d_{0}^{2}\right)^{-2 T_{2}}\right] \\
\cdot f\left(d_{0}\right) \mathrm{d} \tau_{0} \iint_{\mathcal{S}_{i}} f\left(d_{i}\right) \mathrm{d} \tau_{i}, \\
w_{2}=\iint_{\mathcal{S}_{i}} \log \left[\sigma^{2}+A^{2} T_{1}^{2}\left(l^{2}+d_{i}^{2}\right)^{-2 T_{2}}\right] \\
\cdot f\left(d_{i}\right) \mathrm{d} \tau_{i} \iint_{\mathcal{S}_{0}} f\left(d_{0}\right) \mathrm{d} \tau_{0} .
\end{gathered}
$$

Next, we derive the upper bounds under two typical distribution.

1) When Gaussian distribution.

For (35) and (36), let us denote

$$
\begin{aligned}
& w_{1}=\frac{1}{4 \pi^{2} \zeta_{0}^{2} \zeta_{i}^{2}} a_{1}\left(d_{0}\right) \cdot a_{3}, \\
& w_{2}=\frac{1}{4 \pi^{2} \zeta_{0}^{2} \zeta_{i}^{2}} a_{1}\left(d_{i}\right) \cdot a_{2},(i \geq 1)
\end{aligned}
$$

where the terms of $a_{1}\left(d_{0}\right), a_{1}\left(d_{1}\right), a_{2}, a_{3}$ are given in the following,

$$
\begin{gathered}
a_{1}\left(d_{i}\right)=\iint_{\mathcal{S}_{i}}\left(\log \left[\sigma^{2}+A^{2} T_{1}^{2}\left(l^{2}+d_{i}^{2}\right)^{-2 T_{2}}\right]\right) \\
\cdot e^{-\frac{\left(x_{i}-t_{i 1}\right)^{2}+\left(y_{i}-t_{i 2}\right)^{2}}{2 \zeta_{0}^{2}}} \mathrm{~d} \tau_{0},(i \geq 0) .
\end{gathered}
$$

$$
\begin{aligned}
a_{2} & =\iint_{\mathcal{S}_{0}} e^{-\frac{\left(x_{0}-t_{01}\right)^{2}+\left(y_{0}-t_{02}\right)^{2}}{2 \zeta_{0}^{2}}} \mathrm{~d} \tau_{0} \\
& =\int_{0}^{2 \pi} \int_{0}^{u_{0}} e^{-\frac{\rho_{0}^{2}}{2 \zeta_{0}^{2}}} \rho_{0} \mathrm{~d} \rho_{0} \mathrm{~d} \theta_{0}=2 \pi \zeta_{0}^{2}\left(1-e^{-\frac{u_{0}^{2}}{2 \zeta_{0}^{2}}}\right) .
\end{aligned}
$$

Similarly,

$$
a_{3}=\iint_{\mathcal{S}_{i}} e^{-\frac{\left(x_{i}-t_{i 1}\right)^{2}+\left(y_{i}-t_{i 2}\right)^{2}}{2 \zeta_{i}^{2}}} \mathrm{~d} \tau_{i}=2 \pi \zeta_{i}^{2}\left(1-e^{-\frac{u_{i}^{2}}{2 \zeta_{i}^{2}}}\right)
$$




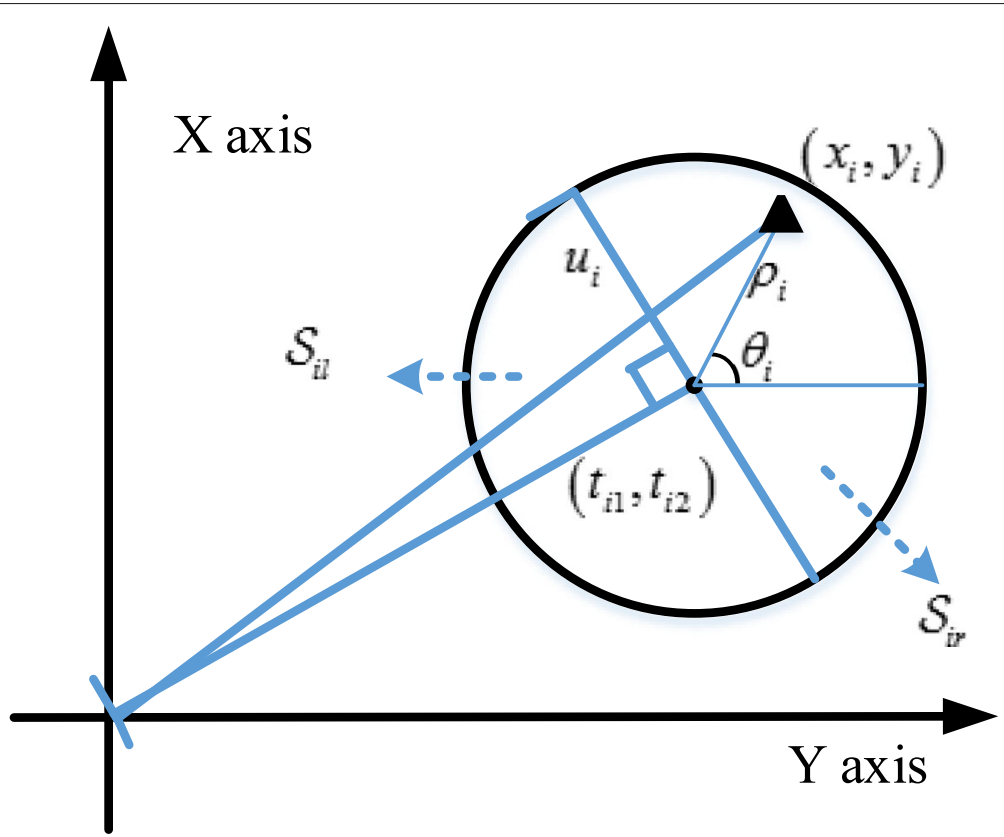

Fig. 8 The sketch map of a typical indoor VLC system and the parameters in Lambertian model

With $T_{2}>0$ in (29), $a_{2}\left(d_{0}\right)$ can be upper-bounded by

$$
\begin{aligned}
& a_{1}\left(d_{0}\right) \stackrel{\left(c_{1}\right)}{=} \iint_{\mathcal{S}_{0 l}}+\iint_{\mathcal{S}_{0 r}} \\
& \stackrel{\left(c_{2}\right)}{\leq} \log \left[\sigma^{2}+A^{2} T_{1}^{2}\left[l^{2}+\left(t_{0}-u_{0}\right)^{2}\right]^{-2 T_{2}}\right] \\
& \cdot \iint_{\mathcal{S}_{0 l}} e^{-\frac{\left(x_{0}-t_{01}\right)^{2}+\left(y_{0}-t_{02}\right)^{2}}{2 \zeta_{0}^{2}}} \mathrm{~d} \tau_{0}+\iint_{\mathcal{S}_{0 r}} e^{-\frac{\left(x_{0}-t_{01}\right)^{2}+\left(y_{0}-t_{02}\right)^{2}}{2 \zeta_{0}^{2}}} \mathrm{~d} \tau_{0} \\
& \cdot \log \left[\sigma^{2}+A^{2} T_{1}^{2}\left[l^{2}+t_{0}^{2}\right]^{-2 T_{2}}\right] \\
& =\frac{a_{2}}{2}\left(\log \left[\sigma^{2}+A^{2} T_{1}^{2}\left[l^{2}+\left(t_{0}-u_{0}\right)^{2}\right]^{-2 T_{2}}\right]+\right. \\
& \left.\log \left[\sigma^{2}+A^{2} T_{1}^{2}\left(l^{2}+t_{0}^{2}\right)^{-2 T_{2}}\right]\right),
\end{aligned}
$$

where the transformation of $\left(c_{1}\right)$ is due to (30) and the approximation of $\left(c_{2}\right)$ is based on (31), (32). Note that $a_{2}$ is calculated in (40).

The term of $a_{1}\left(d_{i}\right)$ can be bounded by

$$
\begin{aligned}
& a_{1}\left(d_{i}\right) \stackrel{\left(\mathcal{C}_{3}\right)}{=} \iint_{\mathcal{S}_{i l}}+\iint_{\mathcal{S}_{i r}} \\
& \stackrel{\left(c_{4}\right)}{\geq} \log \left[\sigma^{2}+A^{2} T_{1}^{2}\left(l^{2}+t_{i}^{2}\right)^{-2 T_{2}}\right] \\
& \cdot \iint_{\mathcal{S}_{i}}^{-\frac{\left(x_{i}-t_{i 1}\right)^{2}+\left(y_{i}-t_{i 2}\right)^{2}}{2 \zeta_{i}^{2}}} \mathrm{~d} \tau_{i}+\iint_{\mathcal{S}_{i}} e^{-\frac{\left(x_{i}-t_{i}\right)^{2}+\left(y_{i}-t_{i 2}\right)^{2}}{2 \zeta_{i}^{2}}} \mathrm{~d} \tau_{i} \\
& \cdot \log \left[\sigma^{2}+A^{2} T_{1}^{2}\left(l^{2}+\left(t_{i}+u_{i}\right)^{2}\right)^{-2 T_{2}}\right] \\
& =\frac{a_{3}}{2}\left(\log \left[\sigma^{2}+A^{2} T_{1}^{2}\left(l^{2}+t_{i}^{2}\right)^{-2 T_{2}}\right]+\right. \\
& \left.\log \left[\sigma^{2}+A^{2} T_{1}^{2}\left(l^{2}+\left(t_{i}+u_{i}\right)^{2}\right)^{-2 T_{2}}\right]\right),
\end{aligned}
$$

where the transformation of $\left(c_{3}\right)$ is due to (30) and the approximation of $\left(c_{4}\right)$ is based on (31), (32). Note that $a_{3}$ is calculated in (41).

Finally, combining (34) with (42), (43), we can obtain

$$
\begin{aligned}
\overline{\mathcal{C}}_{s}\left(h_{B}, h_{E}\right) \leq & \frac{1}{2}\left(w_{1}-w_{2}\right) \\
= & \frac{1}{8 \pi^{2} \zeta_{0}^{2} \zeta_{i}^{2}}\left[a_{1}\left(d_{0}\right) \cdot a_{3}-a_{1}\left(d_{i}\right) \cdot a_{2}\right] \\
\leq & \frac{a_{2} * a_{3}}{16 \pi^{2} \zeta_{0}^{2} \zeta_{i}^{2}} \cdot\left(\log \left[\sigma^{2}+A^{2} T_{1}^{2}\left[l^{2}+\left(t_{0}-u_{0}\right)^{2}\right]^{-2 T_{2}}\right]\right. \\
& +\log \left[\sigma^{2}+A^{2} T_{1}^{2}\left(l^{2}+t_{0}^{2}\right)^{-2 T_{2}}\right]- \\
& \log \left[\sigma^{2}+A^{2} T_{1}^{2}\left(l^{2}+t_{i}^{2}\right)^{-2 T_{2}}\right]- \\
& \frac{\left.\log \left[\sigma^{2}+A^{2} T_{1}^{2}\left(l^{2}+\left(t_{i}+u_{i}\right)^{2}\right)^{-2 T_{2}}\right]\right)}{\left.1-e^{-\frac{u_{0}^{2}}{2 \zeta_{0}^{2}}}\right)\left(1-e^{-\frac{u_{i}^{2}}{2 \zeta_{i}^{2}}}\right)} \mathcal{G}_{1}\left(u_{0}, t_{0}, u_{i}, t_{i}\right) .
\end{aligned}
$$

This completes the proof of (20).

2) When uniform distribution.

For the $i$ th $(i \geq 0)$ receiver,

$$
\iint_{\mathcal{S}_{i}} f\left(d_{i}\right) \mathrm{d} \tau_{i}=\frac{1}{\pi u_{i}^{2}} \int_{0}^{2 \pi} \int_{0}^{u_{i}} \rho_{i} \mathrm{~d} \rho_{i} \mathrm{~d} \theta_{i}=1
$$


Then, using the similar method of proofing (44), we have

$$
\begin{aligned}
& \overline{\mathcal{C}}_{s}\left(h_{B}, h_{E}\right) \leq \frac{1}{2}\left(w_{1}-w_{2}\right) \\
& \leq \iint_{\mathcal{S}_{0}} f\left(d_{0}\right) \mathrm{d} \tau_{0} \\
& \quad \frac{\log \left[\sigma^{2}+A^{2} T_{1}^{2}\left[l^{2}+\left(t_{0}-u_{0}\right)^{2}\right]^{-2 T_{2}}\right]}{\left.\quad \log \left[\sigma^{2}+A^{2} T_{1}^{2}\left(l^{2}+t_{0}^{2}\right)^{-2 T_{2}}\right]\right)} \\
& \quad-\frac{\int_{\mathcal{S}_{i}} f\left(d_{i}\right) \mathrm{d} \tau_{i}}{4}\left(\log \left[\sigma^{2}+A^{2} T_{1}^{2}\left(l^{2}+t_{i}^{2}\right)^{-2 T_{2}}\right]\right. \\
& \left.\quad+\log \left[\sigma^{2}+A^{2} T_{1}^{2}\left(l^{2}+\left(t_{i}+u_{i}\right)^{2}\right)^{-2 T_{2}}\right]\right) \\
& =\frac{1}{4} \mathcal{G}_{1}\left(u_{0}, t_{0}, u_{i}, t_{i}\right) .
\end{aligned}
$$

This completes the proof of (23).

\section{Proof of lower bound (21) and (24)}

We derive the upper bounds based on (12) and (10). Then, we have

$$
\begin{aligned}
& \overline{\mathcal{C}}_{s}\left(h_{B}, h_{E}\right)=\iint_{\mathcal{S}_{0}} \iint_{\mathcal{S}_{i}} \mathcal{C}_{s}\left(d_{0}, d_{i}\right) f\left(d_{0}\right) f\left(d_{i}\right) \mathrm{d} \tau_{0} \mathrm{~d} \tau_{i} \\
& \geq \iint_{\mathcal{S}_{0}} \iint_{\mathcal{S}_{i}} \frac{1}{2} \log \frac{6 h_{B}^{2} A^{2}+3 \pi e \sigma^{2}}{\pi e h_{E}^{2} A^{2}+3 \pi e \sigma^{2}} f\left(d_{0}\right) f\left(d_{i}\right) \mathrm{d} \tau_{0} \mathrm{~d} \tau_{i} \\
& =\int \underbrace{\iint_{\mathcal{S}_{0}} \frac{\log \left(6 h_{B}^{2} A^{2}+3 \pi e \sigma^{2}\right)}{2} f\left(d_{0}\right) \mathrm{d} \tau_{0}}_{b_{1}\left(d_{0}\right)} \underbrace{\iint_{b_{1}} f\left(d_{i}\right) \mathrm{d} \tau_{i}}_{b_{\mathcal{S}_{i}}} \\
& -\underbrace{\iint_{\mathcal{S}_{0}} f\left(d_{0}\right) \mathrm{d} \tau_{0}}_{b_{2}} \int \underbrace{\iint_{b_{1}\left(d_{0}\right) \cdot b_{3}}-b_{2} \cdot b_{1}\left(d_{i}\right) .}_{\int_{\mathcal{S}_{i}} \frac{\log \left(\pi e h_{E}^{2} A^{2}+3 \pi e \sigma^{2}\right)}{2} f\left(d_{i}\right) \mathrm{d} \tau_{i}}
\end{aligned}
$$

1) When Gaussian Distribution. It is easy to know that

$$
b_{2}=a_{2} /\left(2 \pi \zeta_{0}^{2}\right), b_{3}=a_{3} /\left(2 \pi \zeta_{i}^{2}\right)
$$

where $a_{2}, a_{3}$ are in (40) and (41). Applying to the similar method of proofing (44), we have

$$
\begin{aligned}
b_{1}\left(d_{0}\right) \geq & \frac{b_{2}}{4}\left(\log \left[3 \pi e \sigma^{2}+6 A^{2} T_{1}^{2}\left(l^{2}+t_{0}^{2}\right)^{-2 T_{2}}\right]+\right. \\
& \left.\log \left[3 \pi e \sigma^{2}+6 A^{2} T_{1}^{2}\left(l^{2}+\left(t_{0}+u_{0}\right)^{2}\right)^{-2 T_{2}}\right]\right),
\end{aligned}
$$

$$
\begin{aligned}
b_{1}\left(d_{i}\right) \leq & \frac{b_{3}}{4}\left(\log \left[3 \pi e \sigma^{2}+\pi e A^{2} T_{1}^{2}\left(l^{2}+t_{i}^{2}\right)^{-2 T_{2}}\right]+\right. \\
& \left.\log \left[3 \pi e \sigma^{2}+\pi e A^{2} T_{1}^{2}\left(l^{2}+\left(t_{i}-u_{i}\right)^{2}\right)^{-2 T_{2}}\right]\right),
\end{aligned}
$$

Then, the average secrecy capacity is lower-bounded by

$$
\begin{aligned}
& \overline{\mathcal{C}}_{s}\left(h_{B}, h_{E}\right)=\iint_{\mathcal{S}_{0}} \iint_{\mathcal{S}_{i}} \mathcal{C}_{s}\left(d_{0}, d_{i}\right) f\left(d_{0}\right) f\left(d_{i}\right) \mathrm{d} \tau_{0} \mathrm{~d} \tau_{i} \\
& \geq b_{1}\left(d_{0}\right) \cdot b_{3}-b_{2} \cdot b_{1}\left(d_{i}\right) \\
& \geq \frac{\left(1-e^{-\frac{u_{0}^{2}}{2 \zeta_{0}^{2}}}\right)\left(1-e^{-\frac{u_{i}^{2}}{2 \zeta_{i}^{2}}}\right)}{4} \\
& \cdot\left(\log \left[3 \pi e \sigma^{2}+6 A^{2} T_{1}^{2}\left(l^{2}+t_{0}^{2}\right)^{-2 T_{2}}\right]\right. \\
& +\log \left[3 \pi e \sigma^{2}+6 A^{2} T_{1}^{2}\left(l^{2}+\left(t_{0}+u_{0}\right)^{2}\right)^{-2 T_{2}}\right] \\
& -\log \left[3 \pi e \sigma^{2}+\pi e A^{2} T_{1}^{2}\left(l^{2}+t_{i}^{2}\right)^{-2 T_{2}}\right] \\
& \left.-\log \left[3 \pi e \sigma^{2}+\pi e A^{2} T_{1}^{2}\left(l^{2}+\left(t_{i}-u_{i}\right)^{2}\right)^{-2 T_{2}}\right]\right) \\
& \triangleq \frac{\left(1-e^{-\frac{u_{0}^{2}}{2 \zeta_{0}^{2}}}\right)\left(1-e^{-\frac{u_{i}^{2}}{2 \zeta_{i}^{2}}}\right)}{4} \cdot \mathcal{G}_{1}\left(u_{0}, t_{0}, u_{i}, t_{i}\right) \text {. }
\end{aligned}
$$

2) When Uniform Distribution. It is easy to get

$$
\overline{\mathcal{C}}_{s}\left(h_{B}, h_{E}\right) \geq \frac{1}{4} \mathcal{G}_{1}\left(u_{0}, t_{0}, u_{i}, t_{i}\right) .
$$

This completes the proofs of (21) and (24

\section{Proof of lower bounds (22) and (25)}

For simplicity, let us denote the lower bound in (11) as

$$
\mathcal{C}_{L 1}\left(h_{B}, h_{E}\right) \triangleq \frac{p_{1}\left(h_{B}\right)}{2}-p_{2}\left(h_{E}\right)-p_{3},
$$

where the functions of $p_{1}(x), p_{2}(x), p_{3}$ are

$$
\begin{aligned}
& p_{1}(x)=\log \left(1+\frac{2 x^{2} A^{2}}{\pi e \sigma^{2}}\right), \\
& p_{2}(x)=\left(1-2 \mathcal{Q}\left(\frac{\delta+x A}{\sigma}\right)\right) \log \frac{2(x A+\delta)}{\sqrt{2 \pi \sigma^{2}}\left(1-2 \mathcal{Q}\left(\frac{\delta}{\sigma}\right)\right)},
\end{aligned}
$$

$$
p_{3}=\left(\mathcal{Q}\left(\frac{\delta}{\sigma}\right)+\frac{\delta}{\sqrt{2 \pi \sigma^{2}}} e^{-\frac{\delta^{2}}{2 \sigma^{2}}}-\frac{1}{2}\right) .
$$

We can observe that $p_{1}\left(h_{B}\right)$ has no relation with $h_{E}$, while $p_{2}\left(h_{E}\right)$ has no relation with $h_{B}$. Moreover, $p_{3}$ has no 
relation with $h_{B}, h_{E}$. Then, the corresponding $\overline{\mathcal{C}}_{s}\left(h_{B}, h_{E}\right)$ can be lower-bounded as

$$
\begin{aligned}
& \overline{\mathcal{C}}_{s}\left(h_{B}, h_{E}\right) \geq \iint_{\mathcal{S}_{0}} \iint_{\mathcal{S}_{i}} \mathcal{C}_{L 1}\left(h_{B}, h_{E}\right) f\left(d_{0}, d_{i}\right) \mathrm{d} \tau_{0} \mathrm{~d} \tau_{i} \\
& =\iint_{\mathcal{S}_{0}} \iint_{\mathcal{S}_{i}}\left(\frac{p_{1}\left(h_{B}\right)}{2}-p_{2}\left(h_{E}\right)-p_{3}\right) f\left(d_{0}, d_{i}\right) \mathrm{d} \tau_{0} \mathrm{~d} \tau_{i} \\
& =\iint_{\mathcal{S}_{0}} \frac{p_{1}\left(h_{B}\right)}{2} \cdot f\left(d_{0}\right) \mathrm{d} \tau_{0} \iiint_{\mathcal{S}_{i}} f\left(d_{i}\right) \mathrm{d} \tau_{i} \\
& \quad-\iint_{\mathcal{S}_{i}} p_{2}\left(h_{E}\right) \cdot f\left(d_{i}\right) \mathrm{d} \tau_{i} \iint_{\mathcal{S}_{0}} f\left(d_{0}\right) \mathrm{d} \tau_{0} \\
& \quad-p_{3} \iint_{\mathcal{S}_{0}} f\left(d_{0}\right) \mathrm{d} \tau_{0} \iint_{\mathcal{S}_{i}} f\left(d_{i}\right) \mathrm{d} \tau_{i} .
\end{aligned}
$$

Applying to the similar method of proofing (44), (57) can be further approximated as

$$
\begin{aligned}
& \overline{\mathcal{C}}_{s}\left(h_{B}, h_{E}\right) \geq \iint_{\mathcal{S}_{0}} f\left(d_{0}\right) \mathrm{d} \tau_{0} \iiint_{\mathcal{S}_{i}} f\left(d_{i}\right) \mathrm{d} \tau_{i} \\
& \cdot\left[\frac{1}{4} p_{1}\left(h_{0 L}\right)+\frac{1}{4} p_{1}\left(h_{0 R}\right)-\frac{1}{2} p_{2}\left(h_{i L}\right)-\frac{1}{2} p_{2}\left(h_{i R}\right)-p_{3}\right],
\end{aligned}
$$

where

$$
\begin{aligned}
& h_{0 L}=T_{1}\left(l^{2}+t_{0}^{2}\right)^{-T_{2}}, h_{0 R}=T_{1}\left(l^{2}+\left(t_{0}+u_{0}\right)^{2}\right)^{-T_{2}}, \\
& h_{i L}=T_{1}\left(l^{2}+\left(t_{i}-u_{i}\right)^{2}\right)^{-T_{2}}, h_{i R}=T_{1}\left(l^{2}+t_{i}^{2}\right)^{-T_{2}} .
\end{aligned}
$$

When Uniform Distribution, $\iint_{\mathcal{S}_{0}} f\left(d_{0}\right) \mathrm{d} \tau_{0} \iint_{\mathcal{S}_{i}} f\left(d_{i}\right) \mathrm{d} \tau_{i}=1$. When Gaussian Distribution, $\iint_{\mathcal{S}_{0}} f\left(d_{0}\right) \mathrm{d} \tau_{0} \iint_{\mathcal{S}_{i}} f\left(d_{i}\right) \mathrm{d} \tau_{i}=$ $\left(1-e^{-\frac{u_{0}^{2}}{2 \zeta_{0}^{2}}}\right)\left(1-e^{-\frac{u_{i}^{2}}{2 \zeta_{i}^{2}}}\right)$.

This completes the proofs of (22) and (25).

\section{Acknowledgements}

Authors sincerely thank all teachers and graduate students of National Digital Switching System Engineering Technological Research Center for their constant support to the research activity. Especially for Yijun Zhu, Chao Wang.

\section{Authors' contributions}

Project administration and Writing-original draft, Jun Zheng Zhang and Ke Ke. All authors read and approved the final manuscript.

\section{Funding}

The work of this paper was supported in part by the National Key Research and Development Project under Grant 2018YFB1801903, in part by the National Natural Science Foundation of China (NSFC) under Grant 61671477 .
61901524 and in part by the China Postdoctoral Science Foundation under Grant 2019M663477.

\section{Availability of data and materials}

Availability of data and materials.

\section{Competing interests}

The authors declare no conflict of interest.

Received: 28 June 2019 Accepted: 23 April 2020

Published online: 11 May 2020

\section{References}

1. Elgala, H., Mesleh, R., Haas, H.: Indoor optical wireless communication: Potential and state-of-the-art. IEEE Commun. Mag. 2011 (49), 56-62. https://doi.org/10.1109/MCOM.2011.6011734

2. Minh, H. L., Ghassemlooy, Z., O'Brien, D., Faulkner, G.: Indoor Gigabit optical wireless communications: Challenges and possibilities. In: Proc. 12th Int. Conf. Transp. Opt. Netw., pp. 1-6, (2010). https://doi.org/10. 1109/ICTON.2010.5549136

3. Uddin, M. S., Chowdhury, M. Z., Jang, Y. M.: Priority-based resource allocation scheme for visible light communication. In: Proc. 2nd Int. Conf. Ubiquit. Future Netw, pp. 247-250, (2010). https://doi.org/10. 1109/ICUFN.2010.5547195

4. Pan, G., Tang, C., Zhang, X., Li, T., Weng, Y., Chen, Y.: Physicallayer security over non-small-scale fading channels. IEEE Trans. Veh. Technol. 65, 1326-1339 (2016). https://doi.org/10.1109/TVT.2015.2412140

5. Komine, T., Nakagawa, M.: Fundamental analysis for visible-light communication system using LED lights. IEEE Trans. Consum. Electron. 50, 100-107 (2004). https://doi.org/10.1109/TCE.2004.1X277847

6. Nuwanpriya, A., Ho, S. w., Chen, C. S.: Indoor MIMO visible light communications: Novel angle diversity receivers for mobile users. IEEE J. Sel. Areas Commun. 33, 1780-1792 (2015). https://doi.org/10.1109/ JSAC.2015.2432514

7. Zeng, L., O'Brien, D., Minh, H., Faulkner, G., Lee, K., Jung, D., Oh, Y., Won, E. T.: High data rate multiple input multiple output (MIMO) optical wireless communications using white LED lighting. IEEE J. Sel. Areas Commun. 27, 1654-1662 (2009). https://doi.org/10.1109/JSAC.2009. 091215

8. Lapidoth, A., Moser, S., Wigger, M.: On the capacity of free-space optical intensity channels. IEEE Trans. Inf. Theory. 55, 4449-4461 (2009). https:// doi.org/10.1109/TIT.2009.2027522

9. Shannon, C. E.: Communication theory of secrecy systems. Bell Syst. Tech. J. 28, 656-715 (1949). https://doi.org/10.1002/j.1538-7305.1949. tb00928.x

10. Wyner, A. D.: The wire-tap channel. Bell Syst. Tech. J. 54, 1355-1387 (1975). https://doi.org/10.1002/j.1538-7305.1975.tb02040.x

11. Leung-Yan-Cheong, S., Hellman, M.: The Gaussian wire-tap channel. IEEE Trans. Inf. Theory. 24, 451-456 (1978). https://doi.org/10.1109/tit.1978. 1055917

12. Shafiee, S., Liu, N., Ulukus, S.: Towards the secrecy capacity of the Gaussian MIMO wire-tap channel: The 2-2-1 channel. IEEE Trans. Inf. Theory. 55, 4033-4039 (2009). https://doi.org/10.1109/tit.2009.2025549

13. Liu, R., Poor, H.: Secrecy capacity region of a multiple-antenna Gaussian broadcast channel with confidential messages. IEEE Trans. Inf. Theory. 55, 1235-1249 (2009). https://doi.org/10.1109/TIT.2008.2011448

14. Liu, T., Shamai, S.: A note on the secrecy capacity of the multipleantenna wiretap channel. IEEE Trans. Inf. Theory. 55, 2547-2553 (2009). https:// doi.org/10.1109/tit.2009.2018322

15. Khisti, A., Wornell, G. W.: Secure transmission with multiple antennas-Part II: The MIMOME wiretap channel. IEEE Trans. Inf. Theory. 56, 5515-5532 (2010). https://doi.org/10.1109/tit.2010.2068852

16. Oggier, F., VHassibi, B.: The secrecy capacity of the MIMO wiretap channel. IEEE Trans. Inf. Theory. 57, 4961-4972 (2011). https://doi.org/10. 1109/ISIT.2008.4595041

17. Shannon, C. E.: A mathematical theory of communication. Bell Syst. Tech. J. 27, 379-423 (1948). https://doi.org/10.1002/j.1538-7305.1948.tb00917. $x$

18. Farid, A. A., Hranilovic, S.: Capacity bounds for wireless optical intensity channels with Gaussian noise. IEEE Trans. Inf. Theory. 56, 6066-6077 (2010) 
19. Moser, S. M.: Capacity results of an optical intensity channel with inputdependent Gaussian noise. IEEE Trans. Inf. Theory. 58, 207-223 (2012). https://doi.org/10.1109/tit.2010.2080470

20. [12] Wang, J. B., Hu, Q. S., Wang, J., Chen, M., Wang, J. Y.: Tight bounds on channel capacity for dimmable visible light communications. J. Lightw. Technol. 31, 3771-3779 (2013). https://doi.org/10.1109/JLT.2013. 2286088

21. Chaaban, A., Morvan, J., Alouini, M.: Free-space optical communications: Capacity bounds, approximations, and a new sphere-packing perspective. IEEE Trans. Commun. 64, 1176-1191 (2016). https://doi.org/ 10.1109/TCOMM.2016.2524569

22. Jiang, R., Wang, Z., Wang, Q.: A tight upper bound on channel capacity for visible light communications. IEEE Commu. Lett. 20, 97-100 (2016). https://doi.org/10.1109/LCOMM.2015.2497694

23. Mostafa, A., Lampe, L.: Physical-layer security for MISO visible light communication channels. IEEE J. Sel. Areas Commun. 33, 1806-1818 (2015). https://doi.org/10.1109/JSAC.2015.2432513

24. Mukherjee, A.: Secret-key agreement for security in multi-emitter visible light communication systems. IEEE Commun. Lett. 20, 1361-1364 (2016). https://doi.org/10.1109/LCOMM.2016.2558562

25. Shen, H., Deng, Y., Xu, W., Zhao, C.: Secrecy-oriented transmitter optimization for visible light communication systems. IEEE Photon. J. 8 (2016). https://doi.org/10.1109/jphot.2016.2598684

26. Pan, G., Ye, J., Ding, Z:: On Secure VLC Systems With Spatially Random Terminals. IEEE Commun. Lett. 21, 492-495 (2017). https://doi.org/10. 1109/LCOMM.2016.2643632

27. Bloch, M., Barros, J.: Physical-Layer Security: From Information Theory to Security Engineering. Cambridge Univ. Press, Cambridge, U.K. (2011)

28. Cover, T. M., Thomas, J. A.: Elements of Information Theory, 2nd. Wiley, New York (2006)

\section{Publisher's Note}

Springer Nature remains neutral with regard to jurisdictional claims in published maps and institutional affiliations.

\section{Submit your manuscript to a SpringerOpen ${ }^{\circ}$ journal and benefit from:}

- Convenient online submission

- Rigorous peer review

- Open access: articles freely available online

- High visibility within the field

- Retaining the copyright to your article

Submit your next manuscript at $\boldsymbol{\nabla}$ springeropen.com 